\title{
Christian Borde
}

\section{Les deux rêves du commerce maritime au premier $\mathrm{XX}^{\mathrm{e}}$ siècle. Arbitrage social et commercial chez les Armateurs de France (1891-1950)}

L'État serait singulièrement bien inspiré s'il nous laissait, au moins de temps en temps, nous débrouiller tout seuls. Nous n'avons pas toujours besoin, nous, des robes d'avocats ou de juges, de quelque couleur qu'elles soient, pour arriver à nous entendre, pour vivre en bonne intelligence, en un mot pour régler nos différends.

L. Louis-Dreyfus, 1924.

Le Comité Central des Armateurs de France (CCAF) est le syndicat professionnel patronal fondé en 1903 entre les propriétaires de navires de mer. A sa fondation, il regroupait l'ensemble de la profession maritime qui comprenait déjà deux organisations régionales: le Syndicat Marseillais de la Marine Marchande et le Syndicat des Armateurs du Nord, fondés respectivement en 1891 et 1897 . A la veille de la Première guerre mondiale, le CCAF rassemblait la quasi-totalité des autres armateurs au commerce, à la pêche et des services portuaires, en métropole et dans les colonies.

Lorsqu'ils se constituèrent en organisation professionnelle structurée nationalement, les Armateurs de France étaient préoccupés par deux faits nouveaux qui les poussaient à envisager une institutionnalisation de leur action de lobbying. Le premier était d'ordre commercial et consistait à profiter de la création en Europe de nouvelles associations d'armateurs ${ }^{1}$ pour tenter de briser le monopole anglais sur les conditions matérielles du commerce maritime, en particulier la rédaction des connaissements et chartes parties. Comme tous les commerçants, ils pratiquaient à l'occasion et de manière informelle la résolution des conflits à l'amiable c'est-à-dire sans toujours recourir en France aux tribunaux de commerce; le se-

1 Christian Borde, La création du Comité Central des Armateurs de France (13 janvier 1903). Enjeux internationaux et nationaux du lobbying maritime français (1891-1906), dans: Revue d'Histoire Maritime 5 (2006) 221-237. 
cond fait nouveau était la multiplication en France et en Europe des grèves qui agitaient désormais les personnels de tous ordres des compagnies maritimes, aussi bien en mer qu'à terre ${ }^{2}$. Pour cet aspect social de la question, le secteur du shipping français allait être confronté dès 1909 à une innovation gouvernementale sous la forme d'un Conseil permanent d'arbitrage.

Des deux institutions arbitrales dont les naissances vont être rapidement décrites ici, seule la Chambre arbitrale Maritime de Paris subsiste aujourd'hui et on sait que l'arbitrage obligatoire n'existe pas en France. Pourtant chacune correspond à un besoin commun et très utile à toute activité humaine: Pour l'arbitrage social qui fut pour les armateurs efficace mais peu durable, il s'agissait de «régler des conflits qui peuvent naître de l'interprétation des accords antérieurs»; pour l'arbitrage commercial qui fut de son côté peu efficace mais devait se révéler durable, c'était «régler les difficultés nées de l'interprétation ou de l'application des conventions du commerce maritime».

En paraphrasant le titre de la thèse de l'historien Jean-Pierre Hirsch sur les négociants et industriels lillois ${ }^{3}$, ont peut considérer que les armateurs aspiraient à deux rêves en ce qui concerne les pratiques d'arbitrage: d'une part ils rêvaient comme beaucoup d'autres patrons - que leur personnel ne feraient plus jamais la grève et qu'un beau jour l'État les laisserait libre de pouvoir recruter librement leurs équipages en échappant à la contrainte de l'inscription maritime ${ }^{4}$; d'autre part, certains armateurs souhaitaient que le contentieux commercial tant national qu'international puisse être réglé selon les termes mêmes de la profession de foi de la future Chambre arbitrale de 1929, «rapidement et à moindre frais", par un arbitrage qui assurerait également la pertinence des jugements. Mais ce qui est le plus particulier dans la manière qu'eurent les armateurs de considérer l'arbitrage, c'est l'instrumentalisation de l'arbitrage social sur des principes identiques à ceux qui justifiaient l'arbitrage commercial.

2 Ronan Viaud, Le syndicalisme maritime français. Les organisations, les hommes les luttes (1890-1950) (Rennes 2005) 278 (cité comme suit: Viaud, Le syndicalisme).

3 Jean-Pierre Hirsch, Les deux rêves du Commerce, entreprise et institution dans la région Lilloise. 1780-1860 (Paris 1992) 536. L'auteur distinguait deux autres rêves inconciliables des négociants et industriels: d'une part le Laisser-faire et le libre échange, et d'autre part la protection douanière et éventuellement les subventions de l'État.

${ }^{4}$ L'économiste libéral Paul Leroy-Beaulieu décrivait cet espoir le 24 décembre 1902 dans le Journal des Débats, au sujet des équipages en grève à Marseille: "Il ne faut pas que les inscrits maritimes aient le monopole de la navigation sur les navires français. Les armateurs doivent pouvoir recruter comme bon leur semble, à leurs risque et périls, le personnel qu'ils emploient». 


\section{Les concepteurs de l'arbitrage maritime: Paul de Rousiers et Louis Louis-Dreyfus}

L'arbitrage des conflits sociaux fut introduit dans la législation du travail maritime à l'initiative de plusieurs députés socialistes, principalement Joseph Paul-Boncour (1873-1972) passionné par la question sociale et futur ministre du travail de mars à juin 1911. Nous laisserons de côté leur cheminement purement politique pour nous intéresser aux deux représentants du lobby maritime français et promoteurs d'arbitrages que furent Paul de Rousiers (1857-1934) et Louis Louis-Dreyfus ${ }^{5}$ (1867-1940).

\section{La société des "autorités morales» ou les deux arbitrages de Paul de Rousiers}

Même si le syndicalisme marin demeurait un «syndicalisme de métiers»6, l'adhésion de la Fédération Nationale des Syndicats Maritimes à la CGT et la vigueur des conflits faisaient souvent très peur aux armateurs dans la pratique ${ }^{7}$. Le rôle du Comité Central des Armateurs de France fut d'apprivoiser cette phobie patronale en développant face à la question sociale une théorie stratégique qui, tout en disqualifiant les excès du syndicalisme révolutionnaire, ne romprait pas les ponts de la négociation en s'appuyant fortement sur l'appareil juridique contrôlé par le lobby maritime. Son secrétaire général de 1903 à 1934, Paul de Rousiers 8 était un élève de Frédéric Le Play, un écrivain économiste fécond et professeur de Sciences Politiques. Il considérait que les syndicalistes fauteurs de grève faisaient partie de ces «violents qui souhaitent toujours de supprimer tout ce qui s'oppose à leurs désirs, qui, suivant leurs aspirations personnelles, rêvent du Grand soir ou du coup de balai libérateur ${ }^{9}$. Confrontés à ces hommes considérés comme amoraux, P. de Rousiers emprunta directement à Le Play le concept d'«autorité morale». Ces autorités étaient des institutions et pour de Rousiers la notion s'appliquait parfaitement au Comité Central des Armateurs de France. Il suffisait donc que le Syndicat patronal joue le rôle d'arbitre social dévolu aux hommes qui constituaient ces «autorités». Voici comment il les décrivait en 1914:

«Ils se reconnaissent tous à un trait essentiel et caractéristique; ils font régner autour d'eux la paix sociale, repoussent comme une erreur funeste le soi-disant

5 Cette curieuse adoption du nom et du prénom du fondateur comme nouveau patronyme semble relativement rare et peut évidemment prêter à confusion. On la retrouve dans la famille Augustin-Normand, constructeur de navires au Havre.

6 Viaud, Le syndicalisme (n. 2) 37-76; Jean-Pierre Lenhof, Les hommes en mer. De Trafalgar au Vendée Globe (Paris 2005) 313-332.

7 Viaud, Le syndicalisme (n. 2) 115.

8 Antoine Savoye, La professionnalisation des sociologues. L'exemple de Paul de Rousiers (1857-1934), dans: Antoine Savoye, Les débuts de la sociologie empirique (Paris 1994) 203230.

9 Paul de Rousiers, L'élite dans la société moderne. Son rôle (Paris 1914) 173; cité comme suit: Rousiers, L'élite. 
principe de la lutte des classes, et combattent l'antagonisme social sous toutes ses formes. Les doctrines de haine, sous quelque apparence qu'elles se présentent, ne parviennent pas à les séduire; ils savent, en effet, que les sociétés divisées contre elles-mêmes périssent misérablement et que les problèmes sociaux ne se résolvent pas en niant et en supprimant par la force les éléments qui les composent»10.

Paul de Rousiers ne se limita pas à cette vigoureuse dénonciation de la lutte des classes, il envisagea aussi le rôle social très important de ces «autorités» à l'intérieur même de l'élite, qu'il présentaient comme:

«homme ou femme dont l'expérience, la clairvoyance et le bon sens sont universellement prisés. On n'entreprend guère une affaire sans s'aider de ses conseils; on recourt à elle dans les cas difficiles [...] c'est un représentant de l'élite sociale et morale remplissant sa fonction dans son voisinage» 11 .

Un propos qui ne pouvait dans l'esprit de son auteur que séduire le patronat, petit, moyen ou grand puisqu'il décrivait avec sympathie les vertus paternalistes de «certains milieux fermés», et «des personnalités exerçant une influence considérable sur les familles appartenant à ce milieu ${ }^{12}$. On voit comment cette définition de l'élite dirigeante permet de comprendre au plus près la conception des deux types d'arbitrages sociaux que Paul de Rousiers allait tenter de mettre en place de manière concomitante dans le shipping français. Si la résistance aux pressions des employés que le CCAF qualifiait de «défense patronale», était au coeur de l'arbitrage social, c'était par contre la liberté qui présidait à l'arbitrage commercial incarné par la Chambre Arbitrale Maritime de 1929:

«Des liens volontaires rattachent seuls, en dehors de toute contrainte, ceux qui acceptent cette influence et celui qui l'exerce. Et, de même qu'il est le conseiller de ses voisins, il est souvent aussi leur arbitre dans les litiges qui s'élèvent entre eux, particulièrement dans ceux qui relèvent moins d'une contestation juridique que d'une difficulté d'ordre moins précis et plus délicat. Enfin, là où il ne conseille pas expressément, là où il n'arbitre pas, il donne l'exemple, et c'est encore un des aspects importants de sa fonction» 13 .

Mais c'était surtout la «distinction sociale» qui devait régner dans ce domaine. Le choix des arbitres tel qu'il était conçu par le directeur de la Compagnie Générale Transatlantique lors de la constitution de la Chambre arbitrale maritime en 1928-1929 apparaît révélateur de cette application professionnelle des principes leplaysiens:

"Certains des arbitres qui ont été proposés ne sont point des chefs de maison, ni même des chefs de service important de grosses firmes. C'est ainsi que je vois figurer parmi eux des chefs de comptabilité et même certains noms avec indication «de la maison X», sans indication de qualité [...] il me parait qu'il y a à exiger un minimum de standing pour ceux qui sont appelés à jouer un rôle important. [...] Il

10 Ibid. 173-174.

11 Ibid. 171.

12 Ibid. 172.

13 Ibid. 171-172. 
me semble que l'on pourrait poser le principe que pour être arbitre, il faut être véritablement chef de maison ou avoir des connaissances professionnelles résultant d'un brevet comme celui de capitaine au long cours ou de chef mécanicien, ou encore avoir une longue pratique d'affaires, dans une maison dont on ne fait plus partie ${ }^{14}$.

Les «autorités morales» se pensaient aussi garantes du respect des «intérêts», ce que nous appellerions aujourd'hui le lobbying et que Paul de Rousiers évoquait comme «l'intensité de l'action féconde ${ }^{15}$.

Finalement, le respect de l'état de droit était le couronnement de cette conception du monde et de la société dirigés par une élite, capable par les réformes nécessaires d'échapper à la révolution: reconsidérant la place de la morale et du droit dans la société, Paul de Rousiers rappelait que seule l'élite «se souvient que les droits d'autrui sont une force sociale, qu'il est possible, il est vrai, de comprimer temporairement à l'aide d'une force supérieure, mais qu'on rend plus redoutable au fur et à mesure qu'on la comprime davantage» 16 .

\section{Louis Louis-Dreyfus, parrain de la clause compromissoire}

Depuis le XIX siècle, le commerce maritime considérait et revendiquait avec une certaine fierté la situation internationale et fortement spécialisée de son activité dans l'ensemble du commerce ou de l'industrie. Louis Louis-Dreyfus, armateur et député, «parrain» de la loi de 1925, consacrait la clause compromissoire dans l'arbitrage commercial en définissant ainsi la grande diversité des risques maritimes:

«Pertes totales, avaries grosses ou communes, avaries particulières, freintes de route, discussions portant sur la franchise, application des lois étrangères, conflits de législations, interprétation des polices d'assurance, nature des risques, examen de la bonne foi, contrats de réassurances, etc. je ne connais rien de plus délicat en matière commerciale que la discussion des risques de mer, et rien n'est plus voisin $\mathrm{du}$ droit, plus subtil, que cette matière de l'assurance maritime» 17 .

En conséquence, l'existence des multiples contrats de transports et d'assurances et le foisonnement du droit et de la jurisprudence tant nationale qu'internationale étaient souvent difficiles et longs à traiter à l'échelon des tribunaux de commerce.

De plus, au début du XXe siècle, la domination britannique sur le shipping se concrétisa par l'hégémonie du Chamber of Shipping documentary Committee qui rédigeait des chartes-parties pour les lignes de navigation les plus importantes. Créée au Danemark deux ans avant le syndicat patronal français, l'Association des Armateurs Nordiques pensa s'affranchir le la tutelle des Britanniques et déplorait en particulier qu'ils aient été les seuls à trancher les chartes parties et les connais-

14 Archives Nationales du Monde du Travail (ANMT), 52AS 356 le directeur général de la Compagnie Générale Transatlantique au secrétaire général du CCAF, 21 mars 1929.

15 Rousiers, L'élite (n. 9) 175.

16 Ibid.

17 Louis Louis-Dreyfus, La clause compromissoire, conférence faite au $32^{\text {e }}$ Congrès de la Meunerie (Paris 1925) 11. 
sements, imposant à tous leur volonté. Le CCAF pouvait donc dès sa création espérer profiter de cet élan mené par les Scandinaves pour améliorer la position internationale du shipping français. Hélas, force lui fut de constater que cette question apparaissait devoir être tranchée par l'État «dans un sens diamétralement opposé aux intérêts des armateurs ${ }^{18}$. Les entrepreneurs du Nord de la France, regroupés dans le Syndicat des Armateurs du Nord présidé par Adolphe Bordes, plus orientés vers le tramping et la concurrence internationale, s'opposèrent alors à ceux de Marseille surtout préoccupés de trafics coloniaux protégés et dans lequel les compagnies subventionnées avaient une influence prédominante.

Dans un contexte d'unification du droit maritime international, le Comité Central des Armateurs de France s'efforçait d'aplanir ces oppositions. L'une de ses principales activités consistait à fournir à ses adhérents grands ou petits, une information et un conseil juridique de tout premier plan avec l'aide directe des meilleurs experts du droit maritime du temps. Les débats entre professeurs de droit et avocats furent relayés par un conférencier laborieux en la personne du grand armateur et négociant en grains Pierre Louis-Dreyfus qui consacra sa retraite à se passionner pour la clause compromissoire. Il la définissait comme «un engagement que prennent librement les deux parties avant même que la difficulté ne soit née, de la soumettre à l'arbitrage ${ }^{19}$ ce qui avait l'avantage de pouvoir pallier à n'importe quel conflit, même imprévu. La loi du 31 décembre 1925 qu'il avait porté sur les fonds baptismaux du Parlement se heurta à l'hostilité des membres du Barreau mais aussi à celle des Tribunaux de commerce qui, disait Louis-Dreyfus «éprouvent une certaine peine à se voir dessaisir par l'arbitrage d'affaires qui autrement et très normalement seraient allées à eux. Question d'amour propre» 20 .

Dans l'entreprise maritime le recours à des arbitres experts et à la conciliation était à la fois ancien et indispensable. Pour les assurances des navires ou des cargaisons, la validité de la clause compromissoire était convenue dans l'article $332 \mathrm{du}$ Code de Commerce qui définissait la forme du contrat: «La soumission des parties à des arbitres, en cas de contestation, si elle a été convenue». La pratique consistait à confier à des experts spéciaux appelés «dispacheurs» le rôle d'arbitre après qu'ils aient été choisis par les consuls ou par les tribunaux. La «dispache» (de l'italien dispaccio: règlement) consiste à fixer la part contributive de chacun dans les règlements d'avaries.

Louis-Dreyfus déposa une proposition de loi en 1907 qui fut votée à la Chambre en 1908 mais ne passa pas au Sénat, et il fallut attendre 1925 pour qu'elle soit enfin votée par le Parlement. Etienne Clémentel - Fin connaisseur de la pratique commerciale puisqu'il avait été dans sa jeunesse notaire à Riom - présidait alors la Chambre de Commerce Internationale. Après avoir été un brillant ministre innovateur chargé, entre autres, de la marine marchande de novembre 1917 à mai 1919, son soutien ne fut sans doute pas étranger au succès de l'affaire.

18 ANMT, 52AS 370-3, Constitution du CCAF, délibération du SAN, 24 octobre 1902.

19 Louis Louis-Dreyfus, «La clause compromissoire», Le Nord Industriel, 23 mai 1926.

20 Ibid. 


\section{Des rêves à la réalité: l'arbitrage social et le caractère corporatif de l'industrie maritime}

C'est à Marseille que les grèves furent les plus nombreuses et souvent les plus violentes. Cela s'explique par la dureté des pratiques de grévistes précocement syndiqués ${ }^{21}$ et celle des armateurs, d'autant moins conciliants qu'ils disposaient d'un syndicat professionnel particulièrement résistant et indépendant, le Syndicat Marseillais de la Marine Marchande. Ces grèves débutaient souvent par une prise de navire par l'équipage ou encore par l'abandon du bord par celui-ci une fois le rôle constitué22. La paralysie était totale et selon le mot du premier sous-secrétaire d'État à la marine marchande de la IIIe République, Anatole de Monzie, les conséquences de ces grèves étaient souvent catastrophiques: «les primeurs du Tell pourrissaient dans les ports algériens ${ }^{23}$. Aux frais d'immobilisation s'ajoutaient pour les compagnies postales subventionnées des indemnités supplémentaires versées à l'État qui pouvait pousser les armements à la ruine.

\section{Sous la pression des grèves}

Les grandes grèves de Dunkerque en 1900 et de Marseille en 1901 montrèrent au patronat et à l'État la possibilité d'une association des syndicats de dockers avec ceux des marins dans des grèves qui paralyseraient toute activité maritime. Devant cette menace inédite, les armateurs regroupés au sein de leur Syndicat professionnel se solidarisèrent avec les états-majors de leurs navires et décrétèrent un lockout en désarmant tous leurs navires en août 1904. Un ancien président du tribunal de Commerce de Marseille fut finalement choisi comme arbitre souverain: il reconnut le droit pour les syndicats de négocier de nouvelles ententes à condition que les armateurs ne soient plus obligés d'embaucher leurs équipages par l'entremise du syndicat.

La nouvelle grève qui éclata à Marseille en mars 1909 fut principalement provoquée par les modalités d'application de la loi de 1907. Cette loi sur la sécurité et l'hygiène de la navigation maritime, votée le 17 avril 1907 avait en fait vu le jour sous le coup de la poussée syndicale débutée en 1900. Elle constituait pour le gouvernement un renforcement: «du régime tutélaire spécial que l'État a invariablement assuré aux gens de mer et du principe de protection des travailleurs et salariés de toutes catégories qui se retrouve dans tous les actes législatifs fondamentaux édictés en France depuis 25 ans». Son titre 2, consacré à la réglementation du travail à bord des navires, était complété par un second chapitre consacré aux pénali-

21 Viaud, Le syndicalisme (n. 2) 25, signale une «chambre syndicale des matelots français » à Marseille dès 1877.

22 Jean-Pierre Lenhof, Les hommes en mer. De Trafalgar au Vendée Globe (Paris 2005) 313332.

${ }_{23}$ Anatole de Monzie, Les réformes maritimes (Paris 1914) 81; cité comme suit: Monzie, Les réformes. 
tés encourues par les armateurs ${ }^{24}$. Ces derniers se retrouvaient donc en situation de faiblesse, d'autant plus qu'une partie d'entre eux continuaient à demander la suppression de l'inscription maritime et par conséquent du monopole des équipages français.

La loi du 22 juillet 190925 ouvrit la porte à l'instauration dans l'industrie maritime d'un arbitrage qui, sans être obligatoire, devait être particulièrement bien maîtrisé par le gouvernement. Le mot «grève» n'était pas utilisé, ni même celui de «mouvement social»; le législateur lui préférait l'euphémisme suivant: «évènements exceptionnels ayant pour effet d'interrompre temporairement les relations maritimes sous pavillon français». C'était une manière d'étatiser la gravité des conséquences des grèves à répétition, la rupture de continuité territoriale entre la France et la Corse d'une part, et la France et l'Algérie d'autre part. Pour faire pression sur les armateurs, le gouvernement disposait d'une arme très puissante: la suspension par décret de l'exclusif de droit, du monopole concédé auparavant aux armements français pour le trafic avec la Corse ${ }^{26}$. Pour l'Algérie, l'article premier de la loi du 2 avril 1889, susceptible d'être suspendu provisoirement, était particulièrement sibyllin et explicite: «La navigation entre la France et l'Algérie ne pourra s'effectuer que sous pavillon français ${ }^{27}$.

En menaçant de cette mesure d'exception les armateurs, l'État leur enlevait des liaisons très lucratives et exerçait ainsi une pression irrésistible pour l'engagement de négociations. Se sentant trahi, l'armement ne put décourager l'introduction de l'arbitrage. L'instrument de cette stratégie malgré tout très contraignante pour les entrepreneurs fut la création d'un Conseil permanent d'arbitrage qui pouvait être «saisi sans délai des différends d'ordre collectif entre les compagnies de transport et leurs équipages».

Le Décret du 19 mars 191028 - qui ne devait concerner que le secteur du commerce maritime délimité par les lois de 1889 et de 1909, les lignes d'Algérie et de Corse - étendit les compétences de l'arbitrage aux armateurs et équipages de pêche qui disposeraient désormais des mêmes droits que ceux des marins du commerce.

24 Christian Borde, The French State and Safety at Sea. Co-operation and conventions, 1839-1914) (L'Etat français et la sécurité maritime: de la coopération aux conventions internationales, 1839-1914), dans: Bridging Troubled Waters. Conflict and Co-operation in the North Sea Region since 1550, sous la direction de David J. Starkey (Esbjerg 2005) 199-219.

25 Loi du 22 juillet 1909 autorisant le Gouvernement à suspendre temporairement par décret, en cas de circonstances exceptionnelles, l'application de la Loi du 2 avril 1889 et à déroger, dans les mêmes circonstances, à l'article premier de la Loi du 17 juillet 1867, concernant le régime commercial et maritime entre l'Algérie et la Métropole, Journal Officiel du 24 juillet 1909.

26 Par l'application de l'article 4 de la loi du 21 septembre 1793, dite «acte de navigation", Art. 4 : les bâtiments étrangers ne pourront transporter d'un port français à un autre port français, aucune denrées, productions ou marchandises des crû, produit ou manufacturé de France, colonies ou possessions françaises.

27 Loi du 2 avril 1889 relative à la navigation entre la France et l'Algérie, Bulletin officiel de la Marine (Paris 1889) 578.

28 Décret du 19 mars 1910 portant règlement d'administration publique pour l'application de l'art. 2 de la loi du 22 juillet 1909. 
Ce décret qui était en même temps un règlement d'administration publique décrivait en 46 articles un système extraordinairement centralisé mais qui, en même temps, entendait tenir compte du caractère très local de certains litiges. Le conseil siègerait à Paris et il serait composé de juristes arbitres et d'arbitres représentants les employeurs et les employés. Les juristes, choisis parmi «les Conseillers d'État en service ordinaire et parmi les conseillers de la cour de cassation» constituaient la section centrale du Conseil permanent d'arbitrage et siégeaient dans toutes les affaires. Les arbitres représentant employeurs et employés étaient élus pour trois ans et devraient être au moins cinq à siéger dans chaque affaire pour chaque camp. L'assemblée regrouperait ainsi à chaque affaire entre 13 et 23 personnes suivant l'importance géographique des litiges.

Le système ne permettait pas la représentation syndicale mais bien plutôt une division corporative puisque les arbitres des employés étaient répartis en quatre catégories professionnelles:

«1e) les personnes comprises sous la dénomination d'officiers dans l'article 57 de la loi du 15 avril 1898 (modifiant le décret loi de 1852),

$2^{\mathrm{e}}$ ) le personnel du pont;

3e) le personnel des machines,

4 e) les agents du service général».

En raison des difficultés particulières qui agitaient le monde de la «machine», le décret prévoyait même la présence obligatoire dans les arbitres élus de «deux officiers mécaniciens titulaires et deux officiers mécaniciens suppléants».

\section{L'échec de la représentation professionnelle}

Les conséquences du tour de passe-passe peu démocratique qui consistait à élargir le système à la pêche furent catastrophiques. Les armateurs ne participèrent que très peu à l'élection du 31 juillet 1910 et les 75.000 inscrits maritimes pratiquèrent une abstention massive ${ }^{29}$, le taux de participation étant de $2,1 \%$. Un décret de 1911 tenta bien de réorganiser les élections en tenant compte de l'importance du tonnage des entreprises maritimes mais le scrutin de 1a fin 1911 fut un nouvel «échec complet et lamentable» 30 .

C'est surtout par l'organisation de la conciliation locale que l'État tenta par la suite de réguler les grèves de manière contraignante. L'arbitrage renforça d'abord le rôle du Commissaire de l'Inscription maritime qui connaissait aussi bien ses protégés les marins que les armateurs locaux. Ainsi la conciliation pouvait sembler plus aisée: «la question qui divise peut être soumise à l'inscription maritime; Si les deux parties répondent à son appel, l'administration les réunit et s'efforce de les concilier». Le compromis pouvait être signé rapidement, mais en cas d'échec la centralisation reprenait le dessus dans des termes qui jusqu'en 1913 furent assez bureaucratiques. En effet le commissaire de marine devait informer l'ensemble des

29 Monzie, Les réformes (n. 23) 84.

30 Ibid. 
ministères qui s'occupaient des marins et de la navigation maritime, c'est-à-dire pas moins de cinq: Justice, Intérieur, Marine, Commerce et Industrie, Travail et prévoyance sociale. Finalement en 1913, le fonctionnement du conseil fut confié au tout nouveau Secrétariat d'État à la Marine Marchande, dont la nécessité se faisait sentir depuis très longtemps. Même si les séances n’étaient pas publiques, les sentences qui y étaient prononcées pouvaient peser sur l'opinion publique puisqu'elles devaient être publiées par les revues de droit mais surtout par le Journal Officiel où étaient exprimés «le silence ou le refus des parties». Pour le gouvernement et dans certains cas pour les intéressés, employés ou armateurs, le principal avantage de l'arbitrage était contenu dans l'article 38: «Le conseil doit rendre sa sentence dans le plus court délai possible».

Pendant la Première guerre mondiale, l'application des textes de 1909-1910 devint inapplicable en raison de l'impossibilité de procéder aux opérations électorales prévues par le décret. Pour lutter contre les grèves des inscrits, l'État tenta d'abord la manière forte en janvier 1917 lorsque le ministre de l'armement rappela les marins grévistes à leurs devoirs en les menaçant de sanctions. La guerre devenant de plus en plus incertaine, l'État en guerre considéra essentiel «d'aplanir, dans le plus bref délai possible, par voie de conciliation ou d'arbitrage, les différends pouvant s'élever entre les armateurs au long cours et au cabotage et les personnels subalternes du pont et de la machine employés par eux, au sujet de salaires et des autres conditions de travail» et relança la mise en place de la procédure.

\section{Le renforcement de l'Inscription maritime pendant la Première guerre mondiale}

Trois textes de lois se succédèrent de 1917 à 1920 et ne firent que redire en les explicitant les principes de 1909. Qualifiés de «personnel subalterne du pont, de la machine ou du service général » en $1917^{31}$, les marins devenaient en 1919 «le personnel état major du pont et de la machine» mais le Sous-secrétaire d'État des Transports Maritimes et de la Marine Marchande demeurait l'acteur essentiel de la procédure: Il devait être mis au courant des échecs de conciliations «au besoin télégraphiquement »; il présidait en personne la Commission supérieure d'arbitrage qui se tenait dans les locaux de son secrétariat d'État; Enfin, il faisait office d'arbitre départiteur si les arbitres employeurs et employés ne pouvaient se départager.

Les organisations professionnelles nationales d'armateurs et de personnels Comité Central des Armateurs de France et Fédération Nationale des Syndicats maritimes - étaient enfin strictement reconnues. En 1933, le CCAF considérait avec nostalgie cette époque d'union patriotique:

"Il en est résulté tout naturellement l'habitude de procéder à des échanges de vues qui, bien qu'ils fissent ressortir souvent de profondes divisions, pouvaient être - et ont été en fait - le germe des accords passés depuis lors»32.

31 Arrêté du Sous-Secrétaire d'État à la Marine Marchande du 22 décembre 1917, Solution par voie de conciliation des conflits du travail. JO du 24 décembre 1917.

32 ANMT, 52AS 356, note de 1933 sur les conventions collectives. 
La réquisition de la flotte de commerce atteignait $60 \%$ du tonnage français entre 1914 et 1918. Puis, à partir de mars 1918, ce fut l'ensemble de la flotte de commerce au-dessus de 500 t qui fut mise au service de l'État. Par conséquent, les armateurs n'étaient plus maîtres de leurs affrètements ${ }^{33}$. Oubliant cette quasi nationalisation du shipping, le CCAF considérait en 1933 que les armateurs auraient accepté l'idée d'une rémunération plus indifférenciée parce que les navires avaient été utilisés à des trafics qu'ils n'avaient jamais pratiqué auparavant et que leurs équipages avaient été brassés de manière inhabituelle. En fait ce fut le résultat des grèves très dures des années 1919-1921 qui les avaient obligé à céder. La dépréciation du Franc et l'inflation engendra la nécessité d'une négociation permanente au cours de laquelle bon nombre d'armateurs firent preuve comme les syndicats ouvriers d'une mauvaise foi accablante.

\section{Conventions collectives et arbitrages}

Les premiers véritables accords furent signés les 14 et 16 octobre 1920 entre la Fédération Nationale des Syndicats Maritimes et le CCAF après la rupture de l'unité syndicale de mai 192034 . L'épisode de l'Union Navale Paritaire demeure mal connu mais il participait sans doute du rêve corporatiste puisque cette institution regroupa de manière informelle des représentants des armateurs et des représentants des marins. Le 8 juin 1921, les deux parties signèrent un second accord sur les rémunérations et certains détails de l'organisation du service à bord. Pour le CCAF c'était un vrai arbitrage puisqu'il était marqué par une baisse des salaires due à la crise:

«Cette entente est remarquable surtout par le fait qu'en présence de la dépression des frets, qui commençait à s'accentuer dès le milieu de 1920, les représentants des salariés avaient accepté une baisse de salaires. A cette époque, par conséquent, on pouvait dire que le régime de la convention collective donnait des résultats heureux puisqu'il tenait compte à la fois, grâce à la sagesse des représentants des intéressés, des conditions économiques favorables, comme des commissions économiques défavorables ${ }^{35}$.

La loi du 25 septembre 1925 rétablit l'arbitrage considéré par l'État comme un moyen efficace de pression sur les armateurs et les inscrits ${ }^{36}$. Un accord du 19 décembre 1925 comportait un réajustement des salaires et réglait certains détails de service tandis que des accords plus généraux des 26 juin et 29 octobre 1926 établirent des conditions générales d'engagement afin «d'éviter des occasions de conflits». Une fois ces conventions collectives signées, le CCAF allait dès 1926

33 Christian Borde, Les armateurs français au lendemain de la Première Guerre mondiale (1917-1921): Innovation, nationalisation ou américanisation?, dans: Guerre et économie dans l'espace atlantique du $16^{\mathrm{e}}$ au $20^{\mathrm{e}}$ siècle, sous la direction de Silvia Marzagalli, Bruno Marnot (Bordeaux 2006) 83-94.

34 Viaud, Le syndicalisme (n. 2) 85-86.

35 ANMT, 52AS 356 note de 1933 sur les conventions collectives.

36 Viaud, Le syndicalisme (n. 2) 104. 
changer d'attitude et accepter l'arbitrage au jour le jour dans la seule mesure où il s'intégrait dans le suivi de ces conventions. Cette attitude eut un autre effet bénéfique pour le CCAF puisqu'elle renforça la bureaucratisation des syndicalistes ${ }^{37}$ qui n'étaient plus des navigants et dont les principaux dirigeants entrèrent au Conseil Supérieur de la Marine Marchande ${ }^{38}$. Le syndicalisme maritime se réduisait à une remise en cause localisée d'accords patiemment négociés. Après l'élaboration d'un Code du travail maritime par la loi du 13 décembre 1926, l'accord du 18 mai 1929 précisait une échelle de salaires et les conditions générales d'engagements des équipages. Dans le cadre de cette législation, ces conditions étaient susceptibles d'être adaptées à chaque armement ou à chaque variété d'armement puisque l'entreprise maritime recouvrait les différentes pêches et une gamme considérable d'activité de transport. C'est sur ces contradictions et sur l'affaiblissement du mouvement syndical miné par des divisions internes que le CCAF put continuer à jouer au chat et à la souris avec les représentants syndicaux. La convention de 1925 fut remise en cause par la FNSM à partir du 6 août 1930 selon les termes mêmes de l'accord tandis que le CCAF confirmait l'entière solidarité entre les conditions d'engagement et les salaires en raison du grand nombre de primes liées à ces conditions. L'ensemble des entreprises d'armement pouvait par conséquent, en restant relativement solidaires, lutter contre la contagion des revendications et donc des grèves. Elles jouaient à fond le rôle «d'autorités sociales» que leur avaient assigné Frédéric Le Play puis Paul de Rousiers.

Contrairement à l'ensemble de l'économie française frappée seulement en 1932, la marine marchande fut touchée par la crise dès 1929. Grâce au travail de l'arbitrage des années 1920, les armateurs se retrouvaient en situation de force pour imposer, en échange du maintien de l'emploi, des baisses de salaires et leur jugement sur l'arbitrage continua d'être positif:

«Tant que les accords ou conventions collectives ont été en vigueur dans l'industrie des transports maritimes, les conditions qu'ils comportaient ont été généralement observées de part et d'autre, compte tenu des inévitables difficultés d'interprétation qui ont surgi parfois. Il est vraisemblable que, grâce à l'existence de ces accords, des conflits sont pu être évités, les agitateurs de profession ayant moins de facilités pour les faire naître lorsqu'ils se trouvent en présence de conditions précises acceptées de part et d'autre par les groupes les plus représentatifs des armateurs et des équipages ${ }^{39}$.

L'explosion de juin 1936 n'en fut que plus violente et soudaine, rejouant pour les armements les affres de la Belle époque ou du lendemain de la Première guerre Mondiale. Cependant, jusqu'en 1939, l'arbitrage social continua son rôle d'émiettement des revendications des personnels. L'application de la législation sur la conciliation et l'arbitrage fut suspendue par un décret du $1^{\text {er }}$ septembre 1939, et fut

37 Ibid. 95.

38 Ibid. 97.

39 ANMT, 52AS 356 note de 1933 sur les conventions collectives. 
créée une «Commission supérieure de révision des conditions de travail», instituée par l'article 2 du décret du 10 novembre 1939.

\section{La création de la Chambre Arbitrale Maritime en 1929}

C'était la seconde fois que le CCAF créait une institution toute nouvelle à son unique initiative. En effet, il avait suscité directement en 1908, mais dans une assez grande discrétion, la création d'une association de défense des manutentionnaires portuaires: l'Association des employeurs de main d'œuvre dans les ports de France. Elle avait pour objet «l'étude et défense des intérêts communs aux Employeurs de main-d'œuvre dans les ports de France, les progrès techniques et régularité des opérations de manutention dans les ports et la stabilité du personnel». La défense patronale sur mer était ainsi complétée par la défense patronale dans les ports. Mais la discrétion s'imposait sans doute pour que le lien entre les deux syndicats patronaux ne puissent pas faire soupçonner une intention monopolistique. En 1929, au contraire, la création de la Chambre Arbitrale Maritime (CAM) à l'initiative du CCAF fut hautement revendiquée.

«Un certain nombre de groupements d'industriels ou de commerçants intéressés aux affaires maritimes » se rallièrent au CCAF dans ce but ${ }^{40}$. Ce furent d'abord des syndicats professionnels très spécialisés et faciles à convaincre puisque l'arbitrage faisait partie de leur pratique courante: d'abord l'Association des Employeurs de Main d'œuvre dans les ports de France dont le président était le député Hubert Giraud puis le Syndicat des Agents d'Affrètement Maritime présidée par André Sylvain, la Chambre des Négociants commissionnaires et du commerce extérieur présidée par Georges Berger, le Syndicat Central des Négociants Importateurs de Charbons et enfin la Fédération Nationale des Commissionnaires de transports, Transitaires et agents maritimes.

Il fallut ensuite que le CCAF entreprît de convaincre les autres «autorités morales» que l'arbitrage des contentieux par la CAM était supérieur à celui qui est réalisé par les Tribunaux de Commerce. A cet effet Paul de Rousiers vint en exposer les avantages devant l'Union des Chambres de commerce maritimes ${ }^{41}$; il rappela l'intérêt de la clause compromissoire promue par Louis Louis-Dreyfus et souligna la participation entre autres de la Chambre de Commerce de Paris «qui représente une importante clientèle de chargeurs». Le principal avantage de la CAM serait de pouvoir régler les affaires litigieuses sur place dans les ports et non pas à Paris. Enfin, il s'agissait d' «une procédure rapide, peu coûteuse, avec des possibilités données aux parties de choisir leurs arbitres parmi les personnes compétentes ». En définitive, la Chambre Arbitrale se limitait à faciliter la mise en ap-

40 Ibid. Note sur la création d'une chambre arbitrale maritime, 1928 ou 1929.

41 Ibid. Extrait de la séance de l'Union des Chambres de commerce maritimes et des ports français du 9 avril 1929. 
plication de la clause compromissoire facilement insérée dans les contrats grâce à un simple «papillon» de papier.

Le CCAF estimait que la présence des cinq armateurs dans le conseil de la CAM était justifiée "par leur intérêt aux litiges d'ordre maritime.» Cependant, les rapports de force demeuraient bien présents dans ce paradis leplaysien. Ainsi, la Chambre syndicale des Constructeurs de navires se dît très favorable au principe de l'arbitrage et à l'usage de la clause compromissoire mais elle déclara ne pouvoir y participer par crainte que les experts en construction navale soient trop peu nombreux par rapport à ceux des armateurs ce qui, selon elle, aurait pour conséquence que «la liberté de ses adhérents se trouverait entravée» 42 .

Pour les chambres de commerce très liées organiquement aux tribunaux de commerce, l'essentiel était que les entrepreneurs aient toujours la liberté de ne pas insérer la clause compromissoire et d'avoir normalement recours à ces anciennes instances. Le CCAF fut donc obligé de bien souligner que «Les fondateurs de la CAM n'ont aucune hostilité, bien au contraire, contre les tribunaux de commerce qui ne pourront qu'être déchargés et voir ainsi allégée la besogne écrasante qui leur incombe». Le monde du négoce avait horreur de ce qui était obligatoire et lorsque «Une personnalité maritime» - la question parut tellement incongrue que le CCAF ne donna même pas son nom - demanda que l'arbitrage devint une obligation, il lui fut répondu que «le principe de l'obligation ne peut en aucune manière être inscrit en cette matière sous peine d'écarter à priori la plupart des négociants intéressés à un titre quelconque à l'activité maritime».

La Chambre de Commerce de Paris adhéra par principe et celle de Nantes fut acquise dès février 1929 grâce à Julien Roy qui exerçait les fonctions de «dispacheur» et qui rappela à l'occasion que l'encombrement des rôles du Tribunal de Commerce et de la Cour d'appel de Nantes engendrait une attente de deux ans pour qu'un affaire fut traitée. Puis la Chambre de Strasbourg représentée par Moerder, président du Groupement les Usagers du Port de Strasbourg ${ }^{43}$ fut aussi une des premières à adhérer car, en raison du maintien du régime juridique allemand en Alsace, elle avait jugé positive l'expérience des chambres arbitrales. Selon son président elles «rendent les plus grands services, allègent le travail des tribunaux de commerce dont elles complètent heureusement l'activité». Même succès à Dunkerque et à Marseille où les présidents des Chambres arbitrales étaient toujours des présidents sortants des Tribunaux de Commerce. De même, la Chambre du Havre présidée par du Pasquier envoya le président du Tribunal de Commerce pour la représenter.

Mais dans d'autres régions la coexistence avec les tribunaux de commerce posait problème. C'était le cas à Bordeaux ou à La Rochelle où le président Morsch rappelait avec circonspection que «La Chambre Arbitrale maritime ne jouera un rôle vraiment utile que le jour où l'armement français tout entier acceptera d'insérer

42 Ibid. Visite de M. Vergé, secrétaire général de la Chambre syndicale des Constructeurs de navires, 22 novembre 1928.

43 Ibid. séance du conseil de la CAM, 7 mars 1929. 
dans ses contrats la clause compromissoire», ajoutant que dans sa circonscription beaucoup d'entreprises refusaient d'y souscrire. Pour Vasse, président à Fécamp, lorsqu'elle aurait fonctionné «les dissidents y adhéreraient. mais chacun doit demeurer libre de ne pas accepter, dans tous les cas la clause compromissoire». La Chambre de Commerce de Rouen présidée par Faroult était opposée à la CAM pour deux raisons pratiques: l'agenda chargé des présidents qui ne pourraient pas être arbitres et le double emploi avec les compétences des Tribunaux de commerce.

Le conseil d'administration de la CAM se réunît régulièrement entre février 1929 et janvier 1930 en laissant ouverte la liste des arbitres et en tentant de faire mieux connaître ses buts et ses moyens. Mais le succès de ces démarches apparaît très restreint. Dans l'État actuel de nos recherches nous ne connaissons pas le nombre d'arbitrages prononcés mais il semble qu'il ait été très faible. Paul de Rousiers proposa de modifier la formule de recours pour «ménager et prévenir la susceptibilité de certains chargeurs». La modification de la formule de clause compromissoire fut finalement réalisée sur la suggestion d'un des grands armements marseillais, la Société Générale des Transports Maritimes à Vapeur, et, après discussion, elle devint:

«Les litiges nés de l'exécution ou de l'interprétation du présent connaissement seront soumis au tribunal de commerce de ... ou, sur l'accord des parties, à la Chambre Arbitrale Maritime, dans les conditions des statuts et du règlement de cette chambre ${ }^{44}$.

En 1938, Jacques Marchegay, alors secrétaire du CCAF dut constater que le Conseil d'Administration de la Chambre Arbitrale Maritime n'était plus réuni depuis 1930. Il exposa brièvement que la CAM avait régulièrement fonctionné et réglé «un certain nombre de litiges» en évitant soigneusement de chiffrer ce qui fut peut-être une déroute institutionnelle. Son président, Hubert Giraud, président de la Chambre de commerce de Marseille était décédé en cours de mandat sans avoir été remplacé et le décès de Paul de Rousiers en 1934 avait sans doute été fatal à cette première Chambre Arbitrale Maritime. Certains armateurs exprimèrent le regret qu'elle n'ait pas eu une existence plus active et qu'elle ne fût pas connue davantage dans les milieux maritimes, et suggèrent à nouveau de «faire une propagande intense ». En fait en 1931, une Chambre Arbitrale fonctionnait à Marseille tant pour les questions maritimes que pour le commerce et l'industrie en général ${ }^{45}$. Bon nombre des armateurs de ce port étaient depuis longtemps en opposition avec beaucoup des principes et des décisions prises par le CCAF et la crise économique puis la crise sociale de juin 1936 allaient les éloigner encore plus des instances parisiennes.

Toute activité arbitrale semble avoir cessé de février 1939 à décembre 1948. A partir de cette date, une nouvelle institution fut créée qui portait le titre nouveau de «Chambre Arbitrale Maritime de Paris» et qui est toujours en activité de nos jours.

44 Ibid. 28 janvier 1930.

45 Chambre arbitrale des syndicats et groupements commerciaux et industriels de Marseille dite "Chambre arbitrale de Marseille», Liste des arbitres, sections spécialisées, Le Sémaphore de (Marseille 1931). 


\section{Épilogue: arbitrages et corporatisme}

La légalisation des grèves des années 1900 , suivie par celle des procédures d'arbitrage des deux décennies suivantes s'accompagnait après 1930 de l'approfondissement de la crise économique mondiale. Ces trois facteurs eurent tendance à miner la solidarité toute relative qui pouvait exister entre les entreprises d'armement si concurrentes naturellement. Il devint de plus en plus difficile d'unifier les modes de négociation avec les personnels et de maintenir la solidarité entre les armateurs telle qu'elle avait été établie par le système «d'autorités morales» de Paul de Rousiers entre 1903 et le début des années trente. Après 1936, certains, cessant de rêver prirent le temps de négocier avec les syndicats alors que d'autres refusèrent de plus en plus de céder à leurs employés, des capitaines aux soutiers.

La conciliation et l'arbitrage sociaux avaient été conditionnés par la «nature tripartite des relations sociales dans le monde maritime» selon l'expression de l'historien Ronan Viaud: en principe l'État républicain devait servir d'intermédiaire entre les armateurs et les marins et donc il fit souvent fonction d'arbitre pour contrôler l'une et l'autre profession et par conséquent le maintien de l'ordre public. A cet égard, le plus grand arbitrage de la période est constitué par les négociations de juin 1936. Mais à d'autres moments l'État opposa les uns contre les autres au lieu de jouer convenablement son rôle d'arbitre et sa déliquescence à partir de 1934 annonçait des lendemains peu fiables sur le plan des relations sociales.

Dans l'arbitrage commercial, au contraire, l'État n'intervenait plus du tout et pourrait alors se réaliser un autre rêve des armateurs: faire des affaires sans que l'État ni la Justice en apparat n'y ait la moindre part en restant «entre soi».

Pour lutter contre l'étatisme engendré par la crise économique, l'un des rêves nouveaux d'une partie des armateurs fut donc de rassembler les deux acteurs de l'entreprise contre l'État. La théorie des «autorités sociales» et celle de la «destruction de la notion de lutte des classes » pouvaient mener à la conception corporative de la société. Ainsi dès 1929, l'un des buts affichés de la Chambre arbitrale Maritime en 1929 était de «faciliter les rapprochement et par suite une plus complète compréhension entre les différentes branches de l'industrie maritime». D’autres facteurs passéistes concourraient au succès des idées corporatives. On pouvait, comme le faisait Anatole de Monzie en 1914, insister sur le caractère communautaire et rétrograde des acteurs de l'entreprise maritime:

«Pour la plupart, les pêcheurs sont propriétaires de leurs barques. En tout cas, même pour les lointaines campagnes, ils naviguent «à la part», c'est-à-dire qu'ils sont les associés du patron, bien plutôt que ses salariés. De conflits collectifs, de grèves, il n'est pour eux question. D'arbitrage non plus [...] Restent les marins du commerce. Ceux-là sont gens indifférents, sinon fatalistes, qui s'en rapportent volontiers à la Madone ou à l'État-Providence " 46 .

Sur ce désarroi social bien éloigné des utopies de la conciliation sociale et qui accompagnait la crise mais aussi la mutation technologique et des rapports à la mer

46 Monzie, Les réformes (n. 23) 85. 
qui allaient bientôt être complètement bouleversé, devait se construire la seule Corporation qui ait vraiment pris forme dans la France de Vichy, celle des Pêches Maritimes ${ }^{47}$.

\section{Kurzfassung}

Zwei Träume der Seehandelsunternehmer verkörpern sich in der gleichzeitigen Schöpfung zweier Schiedsverfahren, die Konflikte in der Welt der Schifffahrt und der Fischerei in Frankreich am Beginn des 20. Jahrhunderts regeln sollten: Erstens ein Schiedsverfahren, das die Konflikte und Schwierigkeiten der Lohnabschlüsse zwischen den Reedern und ihren Schiffsbesatzungen regeln sollte und das dem Berufsstand von den radikalen Regierungen des frühen 20. Jahrhunderts unter dem Druck der zahlreichen Arbeitskämpfe, die vor dem ersten Weltkrieg in den Reedereien und Häfen ausbrachen, aufgezwängt worden war, und zweitens ein Schiedsverfahren im Handel, das erlaubte, die Zwistigkeiten zwischen Unternehmern schnell und mit geringen Kosten gütlich und unter Achtung der Handelskonventionen zu regeln, falls im Voraus eine „clause compromissoire“ vereinbart worden war.

Paul de Rousiers, Professor für politische Ökonomie, näherte die beiden Schiedsverfahren einander an. Er bezeichnete die Schiedsrichter als „soziale Autorität", ein Begriff, den er direkt dem Denken seines Lehrers Frédéric Le Play, eines der Gründerväter der europäischen Soziologie, entnommen hatte. Von 1903 bis 1934 brachte Paul de Rousiers sein System zur Anwendung. Er handelte in seiner Eigenschaft als Generalsekretär des Zentralkomitees der französischen Reeder, eine Arbeitgebervereinigung, welche die Gesamtheit der Fischerei- und Handelsreeder im Mutterland und den Kolonien zusammenfasste.

Der Beitrag schildert die Art und Weise, in der sich dieses doppelte Konzept entwickelte, das vereinheitlicht und durch die Arbeitgebervereinigung in zweifacher Absicht instrumentalisiert wurde: um eine Alternative zum Klassenkampf zu finden, doch zugleich, um die Solidarität zwischen den Unternehmern zu stärken, die in einer Interessenvertretung vereinigt worden waren, um sie weniger abhängig von der Konsulargerichtsbarkeit sein zu lassen, welche als langsam und kostenintensiv beurteilt wurde. Während die Entwicklung des sozialen Schiedsverfahrens den Sektor der Schifffahrt zu einem korporativen Modell brachte, das durch die Vichy-Regierung auf den Sektor der Fischerei übertragen wurde, blieb das Schiedsverfahren im Handel ein Fehlschlag, obwohl noch heute in Paris die Chambre Arbitrale als Relikt fortbesteht.

47 Viaud, Le syndicalisme (n. 2) 185-192. 


\begin{abstract}
Two dreams of the entrepreneurs of maritime commerce were realized with the simultaneous creation of two courts of arbitration, designed to regulate the conflicts in the field of shipping and fishing in France at the beginning of the 20th $\mathrm{c.:}$ Firstly, an arbitration designed to regulate the conflicts and difficulties to apply the salary agreements between the ship owners and their crew, imposed on the professional community by the radical governments of the early $20^{\text {th }} \mathrm{c}$. under the pressure of numerous strikes which broke out in the shipping companies and the harbors before the first world war. Secondly, a commercial arbitration which allowed regulating the differences between entrepreneurs peacefully and the application of trade conventions quickly and for less money, under the condition that a compromise clause had been concluded in advance. The professeur of political economy Paul de Rousiers assimilated social and commercial arbitration. $\mathrm{He}$ applied to the arbiters the notion of "social authority", borrowed directly from the thinking of his teacher Frédéric Le Play, one of the founding fathers of European sociology. From 1903 to 1934 Paul de Rousiers put his system in application, acting as General Secretary of the Central Committee of the ship-owners of France, a union of entrepreneurs which comprised all owners of fishing and trading ships, in France as well as in the colonies. The article describes the manner in which this double concept developed, unified and exploited by the entrepreneurs' syndicate with a double aim: find an alternative to the class struggle, but also strengthen the solidarity between the entrepreneurs, united in a lobby in order to make them less dependent from the consular justices who were deemed as slow and expensive. While the development of the social arbitrage led the sector of shipping towards a corporative model inaugurated in the sector of fishing by the regime of Vichy, the commercial arbitrage was a failure in spite of the fact that the Chambre Arbitrale de Paris survived as a relic until today.
\end{abstract}

